\title{
The Phenomenon of Word Formation and Composition in Italian Language, Phonetic Aspects and Lexical-Semantic Aspects Related to Them
}

\author{
Arben Skendaj Ph.D. (Professor) \\ University of Tirana, Faculty of Foreign Languages \\ Department of Italian Language \\ Email: arskendaj@hotmail.com \\ Sonila Piri (Professor) \\ University of Tirana, Faculty of Foreign Languages \\ Department of Italian Language \\ Email:sonilap@yahoo.com \\ Irena Ndreu ( Professor ) \\ University of Tirana, Faculty of Foreign Languages \\ Department of Italian Language \\ Email:alen_irena@yahoo.com
}

Doi:10.5901/jesr.2014.v4n4p229

\section{Abstract}

\begin{abstract}
Italian language, as any other language has its mechanisms of word formation and composition related to the enrichment of the vocabulary and the resolution of its lexical problems. It is not easy for Italian language to administer these mechanisms, its structures and microstructures of word formation. It is in a situation which it has to deal with the systems of word formation, which come from Latin, and also that derives from and functions from the nature of Italian. The systems of word-formation, which come from Latin, can be called to be in a "numb" situation, but also in a state that can be used dynamically in Italian language. The phenomenon of word formation stats as a morphological one, but certainly it develops and interferes as a lexical-semantic phenomenon, too. Neologisms in Italian, as in other languages, come from other foreign languages that are called "borrowed words". Italian language, concerning neologisms, that derive other languages, has a special "attitude", compared to other European languages. Some European languages have a more refraction attitude in relation to neologisms and their reaction is very interfering and modifying, even alternating. Whereas, concerning Italian, there is a specific reaction, which comes from the phonetic aspect. Surprisingly, phonetics seems to have a very affective role in lexicology and semantics of a language, in our case of Italian, but that does not result like this.
\end{abstract}

Keywords: word-formation, composition, morphology, lexis, semantics, words, phonetics

Come dalla premessa, prendo in considerazione, per l'enesima volta, problemi relativi al lessico di italiano, ai suoi strati, ai suoi fenomeni di formazione delle parole e come pure situazioni lessicali ereditate dal latino.

A vedere in un primo momento l'italiano paragonato alle altre tre lingue neolatine, francese, spagnolo e portoghese, dal punto di vista fonetico, sembrerebbe, che l'italiano sarebbe dovuto essere rimasto più fedele al latino, e invece no risulterà così. Queste tre lingue neolatine mostrano maggiori aspetti fonetici fedeli verso il latino rispetto all'italiano. Credo, che due sono i fattori più importanti, uno considerando il fattore tempo, cioè il tempo quando queste tre lingue si sono staccate dal latino è molto più lontano del tempo invece quando si è staccato l'italiano col latino. L'italiano sarà stata sicuramente la lingua, che ha accampagnato fino all'ultimo "battito" del latino. E poi il secondo fattore direi, che sarebbe quello geografico, perché le altre tre lingue neolatine non si sono evolute e parlate nello stesso territorio geografico dove si è svolto parlato il latino, invece l'italiano si evoluto e parlato esattamente dove si è parlato il latino. Eppure l'italiano rispetto alle tre lingue neolatine presenta una freschezza maggiore, sembra essere più moderno soprattuttuo foneticamente parlando. Credo, che siano stati appunto i due precedenti fattori ad avere influito a modernizzare e rinfrescare l'italiano, perché il contatto c'è stato fino alla fine dell'esistenza del latino e dopo l'italiano si è trovato doversi modificare per i suoi bisogni e interessi, liberamente dal collegamento dal latino, e lo ha fatto in maniera abbastanza ricreativa, consistente e veloce.

Il francese, lo spagnolo, il portoghese dal momento, che si sono allontanate dal latino si sono "congelate" se così si può dire dal punto di vista fonetico e rispecchiano ancor oggi queste assomiglianze così grandi. 
A ragionare bene, delle tre parti della grammatica di una lingua direi, che la fonetica, la morfologia e la sintassi un po' meno cambiano molto poco rispetto al tempo, già la sintassi mostrerebbe un carattere un po' più elastico, più aperto alle modifiche, sempre, che queste siano necessarie.

Come ho già detto anche in altre sedi l'italiano è una lingua che si è trovata convissuta per 4 secoli con altri suoi dialetti italiani, circa una ventina, cosa molto particolare a confrontare con le altre realtà linguistiche di altre nazioni. Questa situazione l'avrà esposta a un confronto duro esistenziale e concorrenziale con le sue varianti e alternative.

I sistemi di formazione, derivanti dal latino, possiamo chiamarli "in stato congelato", ma pure in stato di sfruttamento in maniera dinamica nella lingua italiana. II fenomeno di formazione nasce come un fenomeno morfologico, ma senza dubbio, che si svolge ed interferisce come un fenomeno poi lessico-semantico.

L'italiano come molte altre lingue ha sempre dei "conti" da fare quanto al lessico, alla formazione delle parole. Questi "conti" sono specifici a seconda delle lingue, alcune lingue hanno problemi minori da risolvere, altre ne hanno un po' di più. Questa differenza deriva da fattori diversi, e soprattutto dallo stato attuale del lessico, dall'appartenenza della lingua alla famiglia di origine e anche dalle pretese, che una lingua eredita ed ha attualmente. La lingua italiana da lingua neolatina, che è sembrerebbe, che avesse gran parte dei suoi problemi, connessi alla formazione delle parole, risolti, invece è solo un'impressione apparente, perché l'italiano non è un latino moderno, effettivamente, è una lingua neolatina, ma è talmente autonoma nei suoi meccanismi di formazione di parole, chr giustamente si considera una lingua molto dinamica da questo aspetto. Ovviamente i problemi della semanticità sono intrinsecamente collegati alla formazione delle parole, alla loro vita lessicale.

I bilanci delle parole in una lingua ovviamente, ufficialmente appartengono ai linguisti, ma ufficiosamente appartengono alla coscienza utilitaria, linguistica della comunità, che parla quella lingua, sono le persone, la gente comune che determina effettivamente la situazione linguisatica, a dire l'ultima parola su un fenomeno, sono le persone parlanti una determinata lingua che stabiliscono cosa modificare, cosa togliere, cosa accettare in una lingua.

Volendo fare un paragone, traendo giustamente simbologia, anche dall'edizione del vocabolario di lingua italiana Zingarelli di qualche anno fa, che sulla cui copertina vi era impressa l'immagine di un vulcano, per illustrare la diversità di situazione lessicale, direi, che se il latino si considerasse come un vulcano spento, l'italiano si considererebbe come un vulcano attivo, la cui situazione non è mai ferma, tranquilla, stabilizzata, ma spesso si verificano eruzioni di diverse proporzioni, la cui "attività eruttiva" cambia anche la "geografia" del suo terreno, e per terreno intendiamo il suo lessico.

Per autonoma si considera relativamente, perché l'italiano col latino ha delle dipendenze assolute, ormai, indiscutibili, sia morfologicamente, lessicalmente che semanticamente.

La lingua italiana dispone ovviamente di una grande capacità di modificare le parole da ogni aspetto grammaticale e semantico.

lo personalmente ho l'impressione " a meno che non mi sbagli", che l'taliano nell'ambito delle lingue europee, pur essendoci anche delle altre grosse lingue di culturali l'italiano sembra, che sia la meno "minacciata" da insidie forestiere del lessico, la più immune lessicalmente parlando. Sembra che le altre lingue si allarmino di più quanto ai neologismi stranieri, e che cerchino subito di "arginare le ferite", e qui cito il francese e il tedesco in particolar modo, rispetto all'inglese e lo spagnolo, invece l'italiano lascia fare, lascia passare tranquillamente, che dopo col tempo, relativamente breve, sistema, "giustizia" i problemi fonetici e lessicali.

L'italiano, foneticamente parlando, sembra rispecchi una "cristallità" di pronuncia, una tonalità, musicalità inviolabile, indelebile, che direi che è in italiano l'aspetto grammaticale e qui mi riferisco alla fonetica, è il più "spietato e crudele" meccanismo linguistico ad adattare la fonetica di un neologismo straniero, a modificarlo secondo le regole, le preferenze e i dettami della lingua italiana sia a livello di scrittura che di pronuncia.

Come sappiamo la formazione delle parole in italiano è un capitolone nel quale troviamo tanti sottocapitoli. Qui possiamo riferire le parole formate con i prefissi, con i suffissi, coi prefissi e suffissi nel contempo, le parole composte, la sostantivazione da verbi ed aggettivi e un'infinità di casi e sottocasi e che non vorrei citare qui, per le intenzioni, dimensioni, cornice e interesse di discussione che mi ero premesso di fare in merito.

La mia preferenza di analisi, in questa discussione, è stata concepita piuttosto sul piano teorico, delle affermazioni e delle costatazioni di natura deduttiva e conclusiva, perché non ho preferito occuparmene di un problema specifico e particolare del lessico e della formazione delle parole, ma rimanere in contesti di una concezione superficiale e generale e di fenomenologia tipica.

Questo capitolone è talmente vasto e intricato, che in una sola discussione di una conferenza non mi sarei mai illuso di poterlo esaurire completamente. Invece rimanendo solo in riflessioni di carattere generale e superficiale, come ho preferito restare, ho pensato di tirare fuori delle problematicità, che aiuterebbero in seguito altri o perchè no anche me stesso a concepire meglio qualche difficoltà lessicale e risolvere più facilmente questi problemi legati alla formazione delle parole. 


\section{La Bibliografia Consultata}

Grande grammatica italiana di consultazione: Tipi di frasi, deissi, formazione delle parole, Lorenzo Renzi, Anna Cardinaletti, Mulino, 1995 - Foreign Language Study.

Grammatica, sintassi, dubbi, Serianni Luca, 2000, Editore Garzanti Libri (collana Le Garzantine)

Linguistica ed educazione linguistica, Einaudi (1978), Monica Berretta

Grande grammatica italiana di consultazione: Tipi di frasi, deissi, formazione delle parole, Lorenzo Renzi, Anna Cardinaletti, Mulino, 1995 - Foreign Language Study.

Grammatica, sintassi, dubbi, Serianni Luca, 2000, Editore Garzanti Libri (collana Le Garzantine)

Linguistica ed educazione linguistica, Einaudi (1978), Monica Berretta

Grande grammatica italiana di consultazione: Tipi di frasi, deissi, formazione delle parole, Lorenzo Renzi, Anna Cardinaletti, Mulino, 1995 - Foreign Language Study.

Grammatica, sintassi, dubbi, Serianni Luca, 2000, Editore Garzanti Libri (collana Le Garzantine)

Linguistica ed educazione linguistica, Einaudi (1978), Monica Berretta

Grande grammatica italiana di consultazione: Tipi di frasi, deissi, formazione delle parole, Lorenzo Renzi, Anna Cardinaletti, Mulino, 1995 - Foreign Language Study.

Grammatica, sintassi, dubbi, Serianni Luca, 2000, Editore Garzanti Libri (collana Le Garzantine)

Linguistica ed educazione linguistica, Einaudi (1978), Monica Berretta

II Sabatini Coletti. Dizionario della Lingua Italiana, Francesco Sabatini, Vittorio Coletti, Rizzoli-Larousse, 2003

Lo Zingarelli, Vocabolario della lingua italiana, Nicola Zingarelli, Zanichelli, Bologna, 1996.

Alberto Sobrero, Introduzione all'italiano contemporaneo (Le strutture), Edizione 2002, Manuali Laterza

Manuale di Semantica 1. Sistemi semiotici John Lyons Laterza, 1980. 